\title{
Aplicação da Endodontia Guiada para localização de canal radicular calcificado apresentando lesão periapical: relato de caso
}

Application of Guided Endodontics to locate the calcified root canal with periapical lesion: case report

Aplicación de endodoncia guiada para la localización del conducto radicular calcificado con lesión periapical: reporte de caso

Thaine Oliveira Lima

ORCID: https://orcid.org/0000-0001-5220-9947

Universidade de São Paulo, Brasil

E-mail: thaineo195@gmail.com

Aurélio de Oliveira Rocha

ORCID: https://orcid.org/0000-0002-9308-2118

Universidade Federal de Santa Catarina, Brasil E-mail: aureliorocha2015@gmail.com

Lucas Menezes dos Anjos

ORCID: https://orcid.org/0000-0001-5100-0789

Universidade Federal de Santa Catarina, Brasil

E-mail: luks anjos@hotmail.com

Rafaela de Menezes dos Anjos Santos

ORCID: https://orcid.org/0000-0002-1517-4527

Universidade Federal de Sergipe, Brasil

E-mail: anjosrafa@outlook.com

Nailson Silva Meneses Júnior

ORCID: https://orcid.org/0000-0003-1384-810X

Universidade de São Paulo, Brasil

E-mail: menesesnailson@gmail.com

Aparecida Emanoelly Sales de Melo ORCID: https://orcid.org/0000-0002-5579-3998

Universidade Tiradentes, Brasil

E-mail:emanoellysales@hotmail.com

Max Dória Costa

ORCID: https://orcid.org/0000-0001-9714-9976

Universidade Tiradentes, Brasil

E-mail:maxdoriacosta@gmail.com

\begin{abstract}
Resumo
Uma solução endodôntica contemporânea para o tratamento endodôntico de canais calcificados é caracterizada pela endodontia guiada. O objetivo deste estudo foi descrever um tratamento de endodontia guiada, sendo está uma técnica que facilita o acesso aos canais radiculares apresentando calcificações pulpares. Paciente do gênero feminino, 40 anos, procurou atendimento em Clínica Odontológica queixando-se de: "dente escurecido". No exame radiográfico, foi observado canal radicular da unidade 21 bastante calcificado, com presença de lesão periapical. Assim, foi indicada a técnica utilizando um guia endodôntico, afim de realizar a localização segura do canal radicular. Após localizado, o canal foi preparado e obturado de forma convencional, dentro das limitações apresentadas pela formação em excesso de dentina de tal unidade. Embora seja uma técnica recém introduzida na literatura, a endodontia guiada garante um menor tempo de trabalho, e tem-se mostrado uma técnica segura e precisa, facilitando o acesso e permitindo um tratamento endodôntico seguro, ágil e com boas condições de previsibilidade.
\end{abstract}

Palavras-chave: Calcificação; Tomografia Computadorizada de Feixe Cônico; Endodontia.

\begin{abstract}
A contemporary endodontic solution for the endodontic treatment of calcified root canals is characterized by guided endodontics. The aim of this study was to describe a guided endodontics treatment, which is a technique that facilitates access to root canals with pulp calcifications. Female patient, 40 years old, sought care at the Dental Clinic complaining of: "darkened tooth". The radiographic examination revealed a highly calcified root canal from unit 21, with the presence of a periapical lesion. Thus, the technique using an
\end{abstract}


endodontic guide was indicated, in order to securely locate the root canal. Once located, the canal was prepared and filled in a conventional manner, within the limitations presented by the excessive formation of dentin in such a unit. Although it is a technique recently introduced in the literature, guided endodontics guarantees a shorter work time, and has been shown to be a safe and precise technique, facilitating access and allowing for a safe, agile and predictable endodontic treatment.

Keywords: Calcification; Cone Beam Computed Tomography; Endodontics.

\section{Resumen}

Una solución endodóntica contemporánea para el tratamiento endodóntico de conductos radiculares calcificados se caracteriza por la endodoncia guiada. El objetivo de este estudio fue describir un tratamiento de endodoncia guiada, que es una técnica que facilita el acceso a conductos radiculares con calcificaciones pulpares. Paciente de sexo femenino, 40 años, que acude a la Clínica Dental quejándose de: "diente oscurecido". El examen radiográfico reveló un conducto radicular altamente calcificado de la unidad 21, con presencia de lesión periapical. Por lo tanto, se indicó la técnica con guía endodóntica, con el fin de ubicar de forma segura el conducto radicular. Una vez localizado, el canal se preparó y rellenó de forma convencional, dentro de las limitaciones que presenta la excesiva formación de dentina en dicha unidad. Aunque es una técnica recientemente introducida en la literatura, la endodoncia guiada garantiza un menor tiempo de trabajo, $\mathrm{y}$ ha demostrado ser una técnica segura y precisa, facilitando el acceso y permitiendo un tratamiento endodóntico seguro, ágil y predecible.

Palabras clave: Calcificación; Tomografía Computarizada de Haz Cónico; Endodoncia.

\section{Introdução}

A calcificação do canal radicular é uma sequela gerada normalmente do traumatismo dentário e pode ocorrer entre 15\%-40\% dos pacientes que sofreram luxação no momento do trauma. Em pacientes idosos, a calcificação pode ser desenvolvida devido uma aposição fisiológica ao longo da vida da dentina secundária e terciária. Além disso, o tratamento ortodôntico pode iniciar a deposição acelerada de dentina secundária. A calcificação pulpar é considerada um sinal de vitalidade da polpa e, a menos que haja o diagnóstico de necrose pulpar, o tratamento do canal radicular não é indicado (Connert et al., 2017; Da Cunha et al., 2009; Mannan et al., 2001). A Associação Americana de Endodontia considerou a terapia endodôntica de canais radiculares calcificados como tendo um alto nível de dificuldade (Sônia et al., 2018; American Association Endodontics, 2013).

Uma solução endodôntica contemporânea para canais calcificados é a utlização de microscópio operatório. Outra alternativa é o uso da tomografia computadorizada cone beam (TCCB), que tem o potencial de auxiliar o operador no aprimoramento das informações para visualização da unidade dental em três dimensões (3D), fornecendo essa representação 3D dos dados radiográficos. O conjunto de dados da TCCB não apenas fornece uma imagem real do dente envolvido, como também permite ao operador detectar a anatomia do canal radicular de forma mais confiável e precisa (Van Der Meer, et al., 2016; Anderson et al., 2018).

Recentemente, um novo método foi introduzido para o tratamento de dentes calcificados, chamado "endodontia guiada" (Krastl et al. 2016; Zehnder et al. 2016). O conceito de endodontia guiada foi descrito pela primeira vez por Krastl et al. (2016) que introduziu esse termo e usou-o em um caso clínico de um incisivo central superior apresentando calcificação pulpar e periodontite apical. Logo apos, Van Der Meer e colaboradores (2016) usaram a endodontia guiada para o tratamento de dentes anteriores superiores com calcificação em três pacientes (Torres et al., 2019).

Para realização dessa técnica um software especial (coDiagnostixTM, Dental Wings Inc., Montreal, Canadá) é utlizado para o alinhamento da TCCB com o scanneamento de superfície, o que permite o planejamento virtual de uma cavidade de acesso ideal. Posteriormente, um guia pode ser produzido por meio de uma impressora 3D. Este guia orienta uma broca minimamente invasiva para a luz do canal radicular calcificado. Um estudo ex vivo ilustrou a alta 
precisão desta técnica, que já foi utilizado com sucesso na prática clínica (Krastl et al., 2016; Connert et al., 2018; Zehnder et al. 2016).

Ao usar um guia impresso em 3D, as chances de dano iatrogênico à raiz são reduzidas e a probabilidade de encontrar o canal radicular é alta, além de reduzir o tempo de tratamento (Krastl et al. 2016; Van Der Meer et al. 2016; Zehnder et al., 2016; Connert et al., 2017, 2018; Torres et al., 2019).

O planejamento virtual e o procedimento de acesso guiado para canais radiculares calcificados ajuda a preservar a estrutura dentária e evita acidentes como desvios e perfurações. Isso pode levar a uma melhora no prognóstico a longo prazo (Van Der Meer et al., 2016). Além disso, o acesso guiado em dentes com calcificação pulpar foi previamente realizado e relatado na literatura como uma técnica segura e previsível (Zehnder et al., 2016). O objetivo do presente estudo é relatar um caso clínico de tratamento endodôntico de um dente com calcificação pulpar por meio do uso da técnica de endodontia guiada.

\section{Metodologia}

O presente estudo configura um relato de caso clínico sobre tratamento endodôntico por meio da utilização da endodontia guiada. O estudo de caso é um método de pesquisa estruturado muito utilizado em diversas áreas da saúde, que pode ser aplicado em distintas situações para contribuir com o conhecimento dos fenômenos individuais ou grupais, bem como difundir o conhecimento da técnica de procedimentos entre os profissionais (Andrade et al., 2017).

Prezando pelos aspectos legais, todas as etapas foram conduzidas em concordância com a Declaração de Helsinque e a Resolução 466/12 do Conselho Nacional de Saúde (CNS). O participante do estudo foi incluido após assinatura no Termo de Consentimento Livre e Esclarecido (TCLE) declarando aceitar participar do estudo e liberando o uso de sua imagem para fins acadêmicos.

\section{Estudo de Caso}

Paciente, sexo feminino, compareceu ao consultório odontológico encaminhado por outro profissional, queixando-se de dente escurecido. Ao exame clínico foi observado que o incisivo central superior esquerdo apresetava alteração de coloração coronária. Ao ser questionado sobre possível histórico de trauma relacionado a unidade em questão, o paciente informou que não recordava qualquer epsódio traumático. Além de negar sintomatologia aos testes de sensibilidade e vitalidade pulpar. No exame radiográfico, foi observado canal radicular da unidade 21 calcificado e presença de lesão periapical (Figura 1). 
Research, Society and Development, v. 10, n. 16, e363101620948, 2021

(CC BY 4.0) | ISSN 2525-3409 | DOI: http://dx.doi.org/10.33448/rsd-v10i16.20948

Figura 1 - Radiografia inicial da unidade 21 indicando canal radicular calcificado.

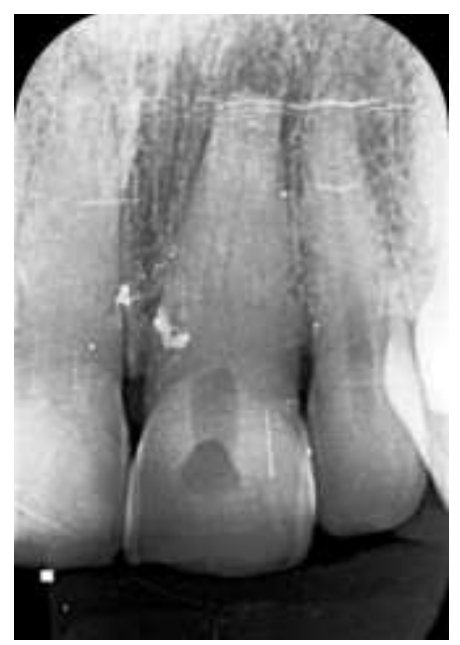

Fonte: Lima, et al. (2021).

Foi solicitada a tomografia computadorizada do tipo cone beam, para melhor avaliação da unidade dentária. Com isso, pode-se delimitar de forma mais precisa a extensão da calcificação pulpar e do canal radicular, a lesão periapical e a quantidade de desgaste feito na tentativa de localização do canal radicular, situações essas que poderiam dificultar o acesso endodôntico e comprometer a unidade dental. Na tomografia, foi observado uma luz tênue de canal radicular em toda extensão da raiz, lesão periapical e um desgaste significativo na porção cervical, porém sem presença de perfuração (Figura 2).

Figura 2 - Corte Sagital da unidade 21 indicando presença de luz tênue do canal radicular.

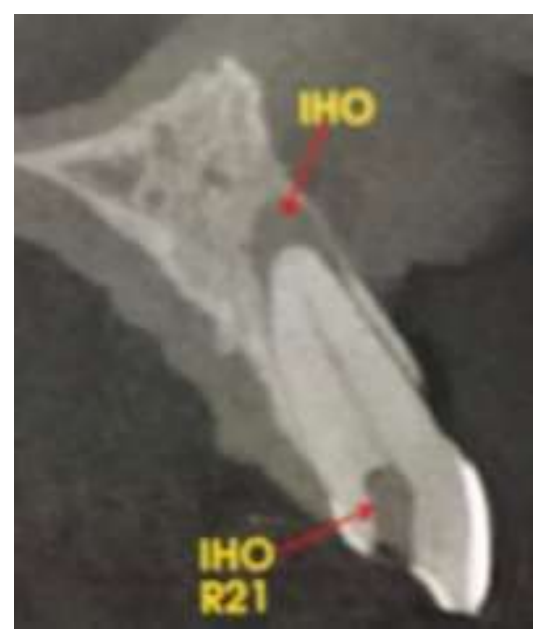

Fonte: Lima, et al. (2021).

Com auxílio da tomografia e do scanneamento intraoral do paciente, foi confeccionado pelo laboratório de imaginologia o guia endodôntico, conhecido como ENDOGUIDE 3D, o qual possibilita o direcionamento para localização do canal radicular pelo cirurgião-dentista (Figura 3). 
Figura 3 - Guia endodôntico confeccionado.

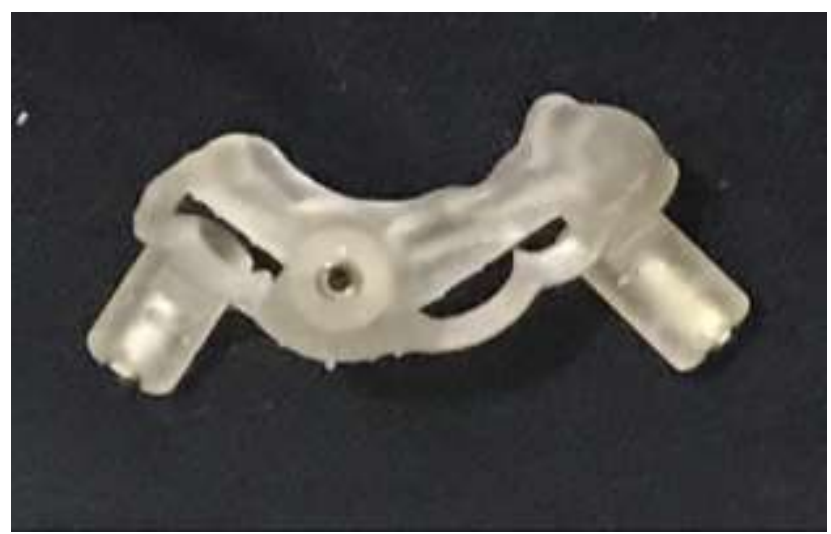

Fonte: Lima, et al. (2021).

Para estabilização do guia, foi necessário realizar a fresagem óssea em dois pontos da maxila. Essa fresagem foi realizada com a broca 103.395 (NEODENT®), sob irrigação constante com soro fisiológico e fixado com parafuros de fixação (NEODENT®).

Com o guia estabilizado, foi realizada a penetração da broca 103.395 (NEODENT®) na abertura coronária convencional previamente feita, removendo a porção de esmalte, atravessando a anilha que dita a quantidade que a broca precisa descer até alcançar a luz do canal, rompendo a calcificação, também sob irrigação constante com soro fisiológico (Figura 4).

Figura 4 - Penetração da broca até o comprimento indicado pela anilha.

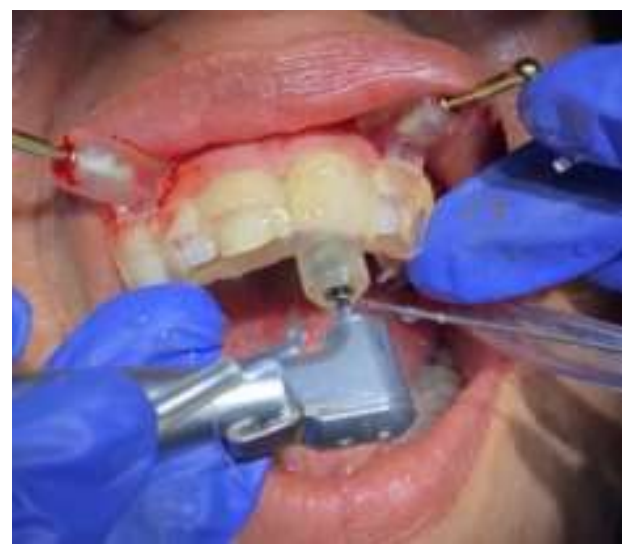

Fonte: Lima, et al. (2021).

Após penetração completa da broca, foi realizado o isolamento absoluto, e localização do canal com lima manual de níquel titánio \#15, C-pilot (DENTSPLY®) e odontometria convencional. Logo após, foi realizada odontometria com auxilio do localizador foraminal (MKLIFE®), constatando um canal de $21 \mathrm{~mm}$ de comprimento no 0.0 obtido (Figura 5). 
Figura 5 - Localização foraminal com localizador eletrônico.

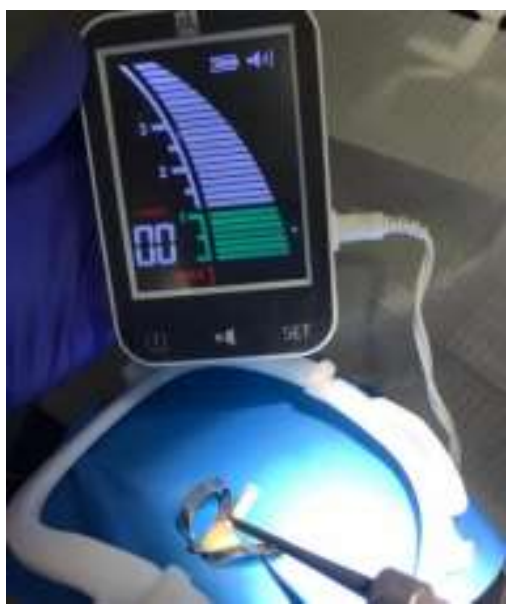

Fonte: Lima, et al. (2021).

Pela presença da calficicação, e dificuldade no corte de dentina durante a instrumentação, o canal radicular foi preparado até o instrumento 30.05 (EASY®), e obturado com cone calibrado e sealer 26 (DENTSPLY®) (Figura 6).

Figura 6 - Radiografia final.

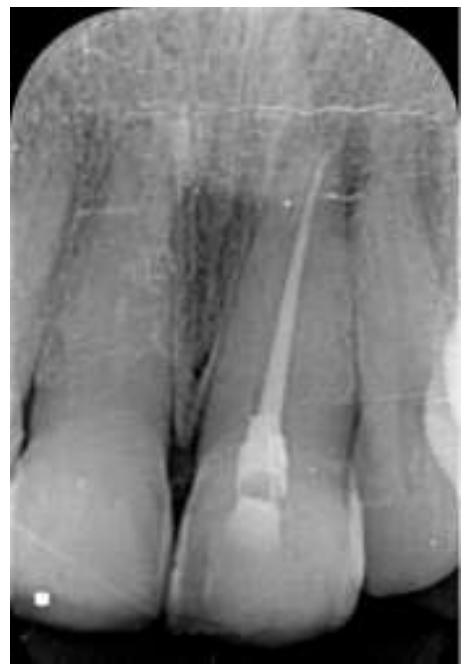

Fonte: Lima, et al. (2021).

\section{Discussão}

O processo de calcificação pulpar pode ocorrer como resultado de trauma dentário, cárie dentária, procedimentos cirúrgicos e raramente, tratamento ortodôntico (McCabe et al., 2016; Fayad et al., 2015; Scheneider et al., 2009). Por outro lado, em idosos, uma aposição ao longo da vida da dentina secundária ou terciária pode resultar em calcificação (Kiefner et al. 2017). Além disso, a resposta aos testes de sensibilidade e ao teste elétrico pode ser ausente, o que dificulta o diagnóstico (Ludlow et al., 2015). Nesses casos, o tratamento endodôntico só deve ser iniciado se o dente apresentar sintomas ou sinais radiográficos de doença periapical (McCabe et al., 2016; Torres et al., 2019; Matherne et al., 2008). No caso apresentado, a paciente não apresentava sintomatologia dolorosa, porém, a presença de lesão periapical analisada no exame radiográfico indicou a necessidade de tratamento endodôntico.

Brocas de haste longa e inserções ultrassonicas são estratégias utilizadas rotineiramente neste tipo de procedimento. No entanto, elas ainda geram um alto risco de falha, mesmo quando associado à ampliação visual com o 
uso de um microscópio cirúrgico (Sônia, et al., 2018; Lara-Mendes et al., 2018). Nesse caso, para evitar falhas e garantir um tratamento com mais segurança, foi optado pela técnica da endodontia guiada.

O conceito de endodontia guiada foi também descrito por Connert et al. (2018) que usaram essa técnica nos incisivos centrais inferiores, onde usaram um pequeno diâmetro $(0,85 \mathrm{~mm})$ em contraste com os tamanhos maiores que foram usados no passado, variando de 1 a 1,5 mm daí o nome "Endodontia Microguiada" (Krastl et al. 2016, Van Der Meer et al. 2016; Patel et al., 2014). No presente caso, um pequeno diametro da broca de tamanho 0,8 mm foi usada devido ao menor tamanho da raiz do incisivo lateral, para conseguir um tratamento minimamente invasivo, mantendo ao mesmo tempo a rigidez quanto possível (Patel et al., 2019; Torres et al., 2019). No caso descrito, se tratando de um incisivo superior, foi utilizada uma broca de diâmetro 1,3mm, o que possibilitou um acesso com preservação de estrutura dentária e com margem para localização do canal radicular.

A endodontia microguida só pode ser usada para dentes com raízes retas ou a parte reta das raízes curvas, o que muitas vezes limita o uso em dentes posteriors, embora já tenha sido descrito na literatura o uso da endodontia guiada em região posterior, inclusive em terceiros molars (Sônia, et al., 2018; Connert et al., 2018). O acesso guiado através de calcificações nas raízes dentes anteriores foi previamente realizado e relatado na literatura com resultados seguros e previsíveis. No presente caso, por se tratar de unidade anterior apresentando canal radicular principal reto, a técnica foi seguramente indicada (Krastl et al., 2016; Connert, et al., 2018).

O tratamento endodôntico guiado pode ser indicado para um acesso mais previsível à secção apical do canal e pode ser mais benéfica para profissionais menos experientes porque elimina a necessidade de um microscópio operacional e permite a máxima preservação da estrutura dental enquanto reduz o tempo de tratamento (Sônia et al., 2018). O custo da técnica desenvolvida é potencialmente mínimo (Van Der Meer et al., 2016).

\section{Conclusão}

Apesar do diagnóstico de calcificação pulpar, a terapia endodôntica só deve ser realizada caso haja indicação com base em exames clínicos e radiográficos analisados de forma minuciosa. Embora o protocolo de tratamento por meio da endodontia guiada exija um maior número de passos, garante um menor tempo de trabalho, e tem-se mostrado uma técnica segura e precisa, facilitando o acesso e permitindo um tratamento endodôntico seguro, ágil e com boa previsibilidade.

Por se tratar de uma tecnologia recente a endodontia guiada necessita de mais estudos clínicos randomizados e revisões sistemáticas, garantindo uma maior robustez de evidência científica e firmando seu uso na prática clínica.

\section{Referências}

Anderson, J., Wealleans, J., \& Ray, J. (2018). Endodontic applications of 3D printing. International endodontic journal, 51(9), $1005-1018$.

Andrade, S. R. D., Ruoff, A. B., Piccoli, T., Schmitt, M. D., Ferreira, A., \& Xavier, A. C. A. (2017). O estudo de caso como método de pesquisa em enfermagem: uma revisão integrativa. Texto \& Contexto-Enfermagem, 26.

Connert, T., Zehnder, M. S., Amato, M., Weiger, R., Kühl, S., \& Krastl, G. (2018). Microguided Endodontics: a method to achieve minimally invasive access cavity preparation and root canal location in mandibular incisors using a novel computer-guided technique. International endodontic journal, 51(2), 247-255.

Connert, T., Zehnder, M. S., Weiger, R., Kühl, S., \& Krastl, G. (2017). Microguided endodontics: accuracy of a miniaturized technique for apically extended access cavity preparation in anterior teeth. Journal of endodontics, 43(5), 787-790.

de Cunha, F. M., de Souza, I. M., \& Monnerat, J. (2009). Pulp canal obliteration subsequent to trauma: perforation management with MTA followed by canal localization and obturation. Braz J Dent Traumatol, 1(2), 64-68.

Endodontics, A. R. (2013). Endodontics colleagues for excellence. Chicago, Illinois: American Association of Endodontists, 1-8. 
Fayad, M. I., Nair, M., Levin, M. D., Benavides, E., Rubinstein, R. A., Barghan, S., ... \& Ruprecht, A. (2015). AAE and AAOMR joint position statement: use of cone beam computed tomography in endodontics 2015 update. Oral surgery, oral medicine, oral pathology and oral radiology, 120(4), 508-512.

Kiefner, P., Connert, T., ElAyouti, A., \& Weiger, R. (2017). Treatment of calcified root canals in elderly people: a clinical study about the accessibility, the time needed and the outcome with a three-year follow-up. Gerodontology, 34(2), 164-170.

Krastl, G., Zehnder, M. S., Connert, T., Weiger, R., \& Kühl, S. (2016). Guided endodontics: a novel treatment approach for teeth with pulp canal calcification and apical pathology. Dental traumatology, 32(3), 240-246.

Lara-Mendes, S. T., Camila de Freitas, M. B., Machado, V. C., \& Santa-Rosa, C. C. (2018). A new approach for minimally invasive access to severely calcified anterior teeth using the guided endodontics technique. Journal of endodontics, 44(10), 1578-1582.

Ludlow, J. B., Timothy, R., Walker, C., Hunter, R., Benavides, E., Samuelson, D. B., \& Scheske, M. J. (2015). Effective dose of dental CBCT-a meta analysis of published data and additional data for nine CBCT units. Dentomaxillofacial Radiology, 44(1), 20140197.

Mannan, G., Smallwood, E. R., \& Gulabivala, K. (2001). Effect of access cavity location and design on degree and distribution of instrumented root canal surface in maxillary anterior teeth. International endodontic journal, 34(3), 176-183.

Matherne, R. P., Angelopoulos, C., Kulild, J. C., \& Tira, D. (2008). Use of cone-beam computed tomography to identify root canal systems in vitro. Journal of endodontics, 34(1), 87-89.

McCabe, P. S. (2006). Avoiding perforations in endodontics. Journal of the Irish Dental Association, 52(3), $139-148$.

Patel, S., Brown, J., Semper, M., Abella, F., \& Mannocci, F. (2019). European Society of Endodontology position statement: Use of cone beam computed tomography in Endodontics: European Society of Endodontology (ESE) developed by. International endodontic journal, 52(12), 16751678 .

Patel, S., Durack, C., Abella, F., Roig, M., Shemesh, H., Lambrechts, P., \& Lemberg, K. (2014). European Society of Endodontology position statement: the use of CBCT in endodontics. International endodontic journal, 47(6), 502-504.

Schneider, D., Marquardt, P., Zwahlen, M., \& Jung, R. E. (2009). A systematic review on the accuracy and the clinical outcome of computer-guided template-based implant dentistry. Clinical oral implants research, 20, 73-86.

Sônia, T. D. O., Camila de Freitas, M. B., Santa-Rosa, C. C., \& Machado, V. C. (2018). Guided endodontic access in maxillary molars using conebeam computed tomography and computer-aided design/computer-aided manufacturing system: a case report. Journal of endodontics, 44(5), 875879 .

Torres, A., Shaheen, E., Lambrechts, P., Politis, C., \& Jacobs, R. (2019). Microguided Endodontics: a case report of a maxillary lateral incisor with pulp canal obliteration and apical periodontitis. International endodontic journal, 52(4), 540-549.

Van Der Meer, W. J., Vissink, A., Ng, Y. L., \& Gulabivala, K. (2016). 3D Computer aided treatment planning in endodontics. Journal of dentistry, 45, 67-72.

Zehnder, M. S., Connert, T., Weiger, R., Krastl, G., \& Kühl, S. (2016). Guided endodontics: accuracy of a novel method for guided access cavity preparation and root canal location. International endodontic journal, 49(10), 966-972. 